\title{
Evaluation of Short-term Mortality of Status Epilepticus and Its Risk Factors
}

Original Article

Journal of Epilepsy Research pISSN 2233-6249 / elSSN 2233-6257

Received December 13, 2014 Accepted February 28, 2015

Corresponding author: Elham Ataei Elham Ataei, Assistant of Neurology, Rasool Akram Hospital, Iran University of Medical Sciences. Tehran, Iran Tel. +98-21-6435-2496

Fax. +98-21-6651-7118

E-mail; eliata1360@yahoo.com

\author{
Mehdi Moghaddasi ${ }^{3}$, Rashin Joodat ${ }^{2}$, Elham Ataei ${ }^{1}$ * \\ ${ }^{1}$ Rasool Akram Hospital Neurology; ${ }^{2}$ Medical Intern, Rasool Akram Hospital, Iran University of Medical Sciences; \\ ${ }^{3}$ Associate Prof. of Neurology, Department of Neurology, Iran University of Medical Sciences, Tehran, Iran
}

\begin{abstract}
Background and Purpose: Status epilepticus (SE) is defined as epileptic seizures of greater than five minutes or more than one seizure within a five minute period without returning to normal consciousness between them. It is a life-threatening condition particularly if treatment is delayed. Previous studies reported age, duration and etiology of SE as primary determinants of mortality.
\end{abstract}

Methods: This prospective cross-sectional study performed on the patients with status epilepticus admitted in Rasoul-e-Akram hospital in Tehran. Patients followed at 30th day after SE to assess their living status.

Results: Sixty-five patients, (56.9\% was male) with 15 to 88 years of age entered the study. Mean duration of SE was 40 minutes and for hospital stay was 7 days. $84.6 \%$ of patients responded to treatment and 11 patients (16.9\%) died within 30 days after SE. Mortality rate in patients with refractory SE was $70 \%$. Mean interval between SE and death was $11.9 \pm 11.7$ days. Age, duration of SE and hospital stay, history of head trauma and neurosurgery were not predictors of mortality. Negative history for epilepsy had significantly higher mortality rate. Anoxic encephalopathy increased the mortality rate and response to treatment decreased it.

Conclusions: Short-term mortality rate of SE was comparable with most of the previous reports Since our hospital has equipped emergency department, resuscitation and primary treatment of SE is usually start soon, percentage of anoxic encephalopathy is decreased and lower mortality rate is expected. We conclude that early treatment by decrease chance of anoxic encephalopathy, has significant role in outcome of SE. (2015;5:13-16)

Key words: Status epilepticus, Mortality rate, Refractory seizure

\section{Introduction}

Status epilepticus (SE) is defined by the International Classification of Epileptic Seizures and by the Epilepsy Foundation of America's Working Group as an epileptic seizure of greater than five minutes or more than one seizure within a five minute period without the person returning to normal consciousness between them. ${ }^{1}$ The seizures can tonic-clonic, absence or complex partial seizures. Status epilepticus is a life-threatening condition particularly if treatment is delayed. ${ }^{2}$

According to a large prospective database compiled through the Greater Richmond Metropolitan Area Status Epilepticus Project (GRMASE), by using the 30-minute definition, 30-day mortality rates for subjects with seizures lasting 10 to 29 minutes was only $2.6 \%$ compared with a $19 \%$ rate in patients diagnosed with SE but who otherwise had comparable clinical features. ${ }^{3}$

As it is uncommon for patients to die during $\mathrm{SE}$, studies generally define SE-related mortality in terms of days lived after resolution of SE. Typically, death rates peak within 30 days after $\mathrm{SE}^{4}$

Status epilepticus may occur in those with a history of epilepsy as well as those with an underlying problem of the brain such as trauma, infections, or strokes. ${ }^{5}$

Status epilepticus is the second common neurologic emergency (after acute stroke) with annual incidence of 10 to 40 cases per 100,000 persons and 10 to $20 \%$ of these patients experience their first seizure. ${ }^{5}$ Apart from duration, an episode that continuous after sufficient dose of Benzodiazepines and at least one other anti-epileptic drug (AED) is called refractory status epilepticus, which is seen in 23 to $43 \%$ of the patients. ${ }^{6}$ 
Previous studies showed $32 \%$ mortality rate in SE episodes lasting for more than one hour versus $2.7 \%$ mortality rate in episodes less than an hour. ${ }^{7}$

Short term mortality rate of refractory SE has been estimated as 16 to $39 \%$. Mortality after refractory SE is three times greater than non-refractory episodes. In the majority of cases, death occurs after SE episode and is related to underlying medical conditions. ${ }^{8}$

After duration and etiology age is the third determinant factor: Age at time of SE predicts a bimodal mortality, with peak rates for the very young and the very old. ${ }^{9}$

Usual mortality rates in adult studies range from $16 \%$ to $25 \%$, although risk steadily increases with advancing age. In the GRMASE study, overall adult mortality was $26 \%$, but $38 \%$ for patients older than 60 years and greater than $50 \%$ in patients older than 80 years. ${ }^{10}$

The overall mortality rates of partial and generalized convulsive SE (GCSE) EEG patterns have not been found to be significantly different. ${ }^{10}$ However, the disassociation of EEG from clinical seizures appears to have a striking effect on mortality. In the Veterans Affairs cooperative study of the treatment of GCSE, patients who have generalized convulsive movements coincident with EEG discharges (overt GCSE) were found to have a 30-day mortality of $26.8 \%$, compared with $64.9 \%$ in those who had no obvious convulsive movements (subtle GCSE). ${ }^{11}$

Mortality rate after SE episode varies in different arias, which can be due to different treatment modalities and geographic conditions. ${ }^{7}$ In the study by Logroscino et al ${ }^{12}$ in 2005, mortality after first SE episode evaluated in the United States and Europe. Incidence and mortality rate of SE was significantly higher in the USA (22\% vs. $10 \%$ mortality rate after exclusion of anoxic encephalopathy cases.)

In the study by Hocker et al $^{13}$ in 2013, 63 non-anoxic refractory SE episodes in 54 adults evaluated. 55 episodes (87.8\%) controlled by anesthetic drugs. Mean duration of admission in hospital was 27.7 days and $31.7 \%$ of patients expired in the hospital. Duration of drug induced coma, cardiac dysrhythmia and pneumonia were predictors of poor prognosis. Patients underwent mechanical ventilation had higher mortality rate.

In the Classen et al ${ }^{14}$ study on 74 patients and 85 episode of $\mathrm{SE}_{\text {, }}$ $21 \%$ of episodes were fatal and $23 \%$ of non-fatal episodes, leaded to decrease functioning of the patient. Old age and acute symptomatic seizure were predictors of mortality.

In a descriptive study by Kulkantrakorn et $\mathrm{al}^{15}$, on 60 patients with $\mathrm{SE}$, most common causes include AED withdrawal and ischemic stroke.
$13 \%$ of patient had refractory SE and 25\% expired. Factors associated with higher mortality rate included advanced age, longer duration of seizure and coma.

Although some clinical series have suggested $s$ decrease in mortality in SE patients in the recent years, the Rochester Epidemiologic Study, ${ }^{4}$ which calculated incidence and case-fatality rates (CFR) over 30 years (1955 to 1984), found that the 30-day CFR remained stable. However, incidence rates increased as a result of greater numbers of elderly patients and survivors of cardiac arrest with anoxic etiology for SE. Thus, despite the better treatment options and trends toward recommending more aggressive ICU management for SE, it appears that these improvements in patient care are merely matching the increased proportion of high-mortality SE cases.

The aim of the present study is to evaluate 30-day mortality rate and prognostic factors in patients with $\mathrm{SE}$, who are admitted in Rasoul-e-Akram hospital, Tehran, Iran.

\section{Methods}

This study has been designed as a prospective cross-sectional study. Study population is all the patients with status epilepticus that visited emergency department and admitted in Rasoul-e-Akram hospital in Tehran, Iran, from October 2013 to December 2014.

Inclusion criteria are: consent of patient or his/her family to enter the study, more than 15 years of age. Patients with other forms of SE (other than tonic-clonic SE) did not enter the study.

SE is confirmed by clinical judgment and EEG findings; as well as witness report. Patients with suspicious history regarding status seizures or whom we couldn't judge about the exact duration of seizure were excluded. A check list is made for each patient and data such as age, gender, history of epilepsy or SE, history of surgery, presence of anoxic encephalopathy, duration of SE and duration of admission are recorded.

\section{Treatment protocol}

Initial assessment: ensuring adequate ventilation, oxygenation, blood pressure, intubation if low oxygen saturation and labored breathing; inserting intravenous line, administration of glucose in appropriate circumstances and for rare cases in Iran thiamine if history of alcoholism is obtained. Send blood samples for toxicology and AED level assessment.

Immediate suppression of convulsions. diazepam 2-4 mg/min IV to a total dose of 10-15 mg with monitoring of Vital signs 
Initiation or reloading with anticonvulsants. slow infusion of phenytoin $15-20 \mathrm{mg} / \mathrm{kg}$ (25-50 mg/min, diluted in normal saline)

General anesthetic doses of medication for SE: if convulsions continued, the patient is considered to have refractory SE (ten patients); then Midazolam $0.2 \mathrm{mg} / \mathrm{kg}$ loading dose followed by infusion at 0.1 $0.4 \mathrm{mg} / \mathrm{kg} / \mathrm{h}$ or Propofol $2 \mathrm{mg} / \mathrm{kg} / \mathrm{h}$ were administrated; and if convulsions persist after several hours, Valproate or Phenobarbital 10 $\mathrm{mg} / \mathrm{min}$ to total dose of $20 \mathrm{mg} / \mathrm{kg}$ were administrated intravenously. Alternatively, Carbamazepine or Levetiracetam were administered by nasogastric tube if bowel activity present, and if convulsions persist, neuromuscular paralysis was done with....

All the patients admitted to intensive care unit after suppression of convulsion in emergency department.

Hypoxic encephalopathy was suspected if patient had prolonged loss of consciousness for more than time we expect as post ictal phase, without any other cause. The diagnosis was confirmed if parkinsonism signs with cognitive impairment or abnormal movements (myoclonus or choreathetosis) which didn't exist before SE, develop; laboratory reveal acidosis in blood gas analysis, neuroimaging show loss of distinction between cerebral gray and white matter and diffuse signal changes specially in striatal regions, and generalized slow waves seen in electroencephalogram.

All the patients received a phone - call at 30th day after SE to assess their living status.

Data analysis is done by SPSS version 18 and descriptive analysis such as mean, standard deviation and range are calculated. Studentt-test is used for quantitative analysis and Chi-2 test is used for analysis of qualitative variants.

\section{Results}

Sixty-five patients, who referred to emergency department of Rasoul-e-Akram hospital with SE, treated and admitted in neurology ward, entered the study with informed consent. 37 patients (56.9\%) were male. Patients had 15 to 88 years of age with mean of 44.6 years.

Mean duration of SE in the cases was 40 minutes and mean duration of hospital stay was 7 days. $21.5 \%$ and $6.2 \%$ of patients had history of head trauma and neurosurgery, respectively. About half of them (47.7\%) were previously diagnosed with epilepsy and $12.3 \%$ had experienced previous episode of SE. Anoxic encephalopathy was seen in $13.8 \%$ of the cases (Table 1 ).

Fifty-five patients $(84.6 \%$ ) responded to treatment (ten other pa- tients had refractory SE) and 11 patients (16.9\%) died within 30 years after SE. Mortality rate in patients with refractory SE was 70\%. Mean interval between SE and death was $11.9 \pm 11.7$ days. Mortality rate did not differ between the two genders ( $p=0.18$ ).

Age, duration of SE and duration of hospital stay were not significant predictors of mortality in our patients $(p=0.08$. 0.65 and 0.41 , respectively).

Mortality in patients with history of head trauma and neurosurgery was equal with those with negative history $(p=0.1$ and 0.99 , respectively).

Patients with negative history for epilepsy had significantly higher mortality rate. $(p<0.0001$, Odds Ratio $=7.7)$ But mortality rate in those with positive and negative history for previous SE episode was not different. ( $p=0.99)$

Anoxic encephalopathy significantly increased the mortality rate. $(p<0.0001, \mathrm{OR}=20.4)$ and desirable response to treatment sig-

Table 1. Basic characteristics of the patients

\begin{tabular}{ll}
\hline Value & $37(56.9 \%) / 28(43.1 \%)$ \\
\hline Male/female & $44.69 \pm 20.7$ \\
Age (year) & DM $3(4.6 \%)$ \\
Underline disease & HTN $4(6.2 \%)$ \\
& CKD $3(4.6 \%)$ \\
& $14(21.5 \%)$ \\
Head trauma history & $4(6.2 \%)$ \\
Brain surgery history & $31(47.7 \%)$ \\
Epilepsy history & $8(12.3 \%)$ \\
Status Epilepticus (SE) history & $9(13.8 \%)$ \\
Anoxic Encephalopathy history &
\end{tabular}

Table 2. Factors influencing mortality rate of SE

\begin{tabular}{ll}
\hline Variable & \multicolumn{1}{c}{$p$ value } \\
\hline Sex $(\mathrm{f} / \mathrm{m})$ & 0.054 \\
Age & 0.064 \\
History of head trauma $(+/-)$ & 0.1 \\
History of neurosurgery $(+/-)$ & 0.99 \\
History of previous SE $(+/-)$ & 0.99 \\
Epilepsy history $(+/-)$ & 0.997 \\
Response to treatment $(+/-)$ & $0.018^{*}$ \\
Anoxic encephalopathy $(+/-)$ & $P<0.0001$ \\
Duration of SE & 0.65 \\
Duration of hospital stay & 0.41
\end{tabular}

*expresses significant influence of response to treatment on the mortality rate of SE. 
nificantly decreased it $(p<0.0001, \mathrm{OR}=29.7)$ (Table 2).

\section{Discussion}

Status epilepticus, as the second most common neurologic emergency, has high risk of mortality and morbidity. Previous studied estimated 16 to $39 \%$ mortality rate for refractory SE, which is tree time greater than non-refractory SE. ${ }^{8}$

In this study, total mortality rate was $16.9 \%$ and slightly higher in females; however, the difference was not statistically significant. In the study by Koubeissi et al, female patients had significantly greater mortality rate. $^{16}$

Mortality rate in first month after SE reported $31.7 \%$ by Hocker et $\mathrm{al}^{13}$ and $21 \%$ by Classen. ${ }^{14}$ Another study performed in 2005 , reported $10 \%$ mortality rate for $\mathrm{SE}^{12}$ In pediatric age group, 14.6\% mortality rate was reported ${ }^{11}$ and in study by Kubeissi in the USA, in-hospital mortality rate was $3.45 \%{ }^{16}$

In this study short-term mortality rate of SE was comparable with most of the previous reports and even lower than some others. ${ }^{13,14}$ Since Rasoul-e-Akram is a referral hospital, it has well-equipped emergency department, resuscitation and primary treatment of $S E$ is usually started very soon and metabolic causes for SE and electrolyte imbalances are corrected rapidly. Thus, percentage of patients with anoxic encephalopathy is decreased and less mortality rate, compared to some other hospitals, is expected.

Previous studies reported long-term mechanical ventilation; ${ }^{13}$ advanced age ${ }_{i}^{16,17}$ longer duration of SE; ${ }_{i}^{4,17}$ coma at presentation; ${ }_{i}^{17}$ female sex, hypoxic-ischemic brain injury, higher rate of comorbidities, ${ }^{14}$ generalized myoclonic seizure, acute symptomatic etiology for seizure ${ }^{4,12}$ and refractory $\mathrm{SE}$, as predictors of higher mortality. However, in our study, age, sex, duration of SE, duration of hospital stay, history of head trauma or neurosurgery and previous episode of SE were not associated with mortality; but mortality rate was significantly less in patients who responded to treatment; which is comparable with Saenz's study. ${ }^{11}$ Moreover, patients with anoxic encephalopathy and those with negative history for epilepsy had higher mortality rate.

Furthermore, from another aspect we found that patients with negative history for epilepsy had significant higher mortality rate that a hypothesis is refractory SE and it's sequels are maybe because of an underlying cause as drug poisoning or other causes that our source of history is not aware of that and can't be demonstrated in routine toxicity lab tests.

\section{References}

1. Gastaut H. Classification of status epilepticus. In: Delgado-Escueta A, Wasterlain C, Treiman D, Porter R, editors., eds. Status epilepticus: Mechanisms of brain damage and treatment. Raven Press, 1983;15-35.

2. Al-Mufti F, Claassen J. Neurocritical care: status epilepticus review. Critical care clin 2014;30:751-64.

3. DeLorenzo RJ, Garnett LK, Towne AR, et al. Comparison of status epilepticus with prolonged seizure episodes lasting from 10 to 29 minutes. Epilepsia 1999:40:164-9.

4. Logroscino G, Hesdorffer DC, Cascino GD, Annegers JF, Bagiella E, Hauser WA.. Long-term mortality after a first episode of status epilepticus. Neurology 2002;58:537-41.

5. Trinka E, Höfler J, Zerbs A. Causes of status epilepticus. Epilepsia 2012;53(Suppl 4):127-38.

6. Rossetti $\mathrm{AO}$, Lowenstein $\mathrm{DH}$. Management of refractory status epilepticus in adults: still more questions than answers. Lancet Neurol 2011;10:922-30.

7. Neligan A, Shorvon SD. Prognostic factors, morbidity and mortality in tonic-clonic status epilepticus: a review. Epilepsy Res 2011;93:1-10.

8. Rossetti AO, Lowenstein DH. Management of refractory status epilepticus in adults: still more questions than answers. Lancet Neurol 2011;10:922-30.

9. Hauser WA. Status epilepticus: Epidemiologic considerations. Neurology 1990;40(5 suppl 2):9-13.

10. Towne AR, Pellock JM, Ko D, DeLorenzo RJ. Determinants of mortality in status epilepticus. Epilepsia 1994;35:27-34.

11. Treiman DM, Meyers PD, Walton NY, et al. A comparison of four treatments for generalized convulsive status epilepticus. Veterans Affairs Status Epilepticus Cooperative Study Group. N Eng/ J Med 1998;339: 792-8.

12. Logroscino G, Hesdorffer DC, Cascino G, et al. Mortality after a first episode of status epilepticus in the United States and Europe. Epilepsia 2005;46(Suppl 11):46-8.

13. Hocker SE, Britton JW, Mandrekar JN, Wijdicks EF, Rabinstein AA. Predictors of outcome in refractory status epilepticus. JAMA Neurol 2013;70:72-7.

14. J. Claassen J, Lokin JK, Fitzsimmons BF, Mendelsohn FA, Mayer SA. Predictors of functional disability and mortality after status epilepticus. Neurology 2002;58:139-42.

15. Kulkantrakorn K, Moonman W. Treatment outcome of status epilepticus in Thammasat University Hospital, Thailand. Neurology Asia 2014;19: 257-62.

16. Koubeissi M, Alshekhlee A. In-hospital mortality of generalized convulsive status epilepticus. Neurology 2007;69:886-93.

17. Ristić AJ, Sokić DV, Trajković G, et al. Long-term survival in patients with status epilepticus: a tertiary referral center study. Epilepsia 2010; 51:57-61. 